\title{
Electronic communication in ethics committees: experience and challenges
}

Arnold R Eiser, Stanley G Schade, Lisa Anderson-Shaw and Timothy Murphy University of Illinois at Chicago, USA

\begin{abstract}
Experience with electronic communication in ethics committees at two hospitals is reviewed and discussed. A listserver of ethics committee members transmitted a synopsis of the ethics consultation shortly after the consultation was initiated. Committee comments were sometimes incorporated into the recommendations. This input proved to be most useful in unusual cases where additional, diverse inputs were informative. Efforts to ensure confidentiality are vital to this approach. They include not naming the patient in the e-mail, requiring a password for access to the listserver, and possibly encryption. How this electronic communication process alters group interactions in ethics committees is a fruitful area for future investigation.

(Fournal of Medical Ethics 2001;27 supp1 I:i30-i32)
\end{abstract}

Keywords: Clinical ethics committees; electronic communication; confidentiality

\section{Introduction}

The core process for a health care organisation's ethics committee is creating informed dialogue on the ethical implications of clinical care decisions and organisational policies. While the policy issues can be addressed at regularly scheduled meetings, ethical interpretations concerning specific patient care matters require prompt, often urgent, response. While this is commonly completed in a successful fashion by the ethics consultant or ethics consultation team, there arise a number of cases where the wider input of the ethics committee would provide additional insights and benefits. In the past some institutions' committees responded by calling urgent ad hoc meetings that were often difficult to convene at short notice. Moreover, when this occurs, it often diverts committee members from other responsibilities.

The advent of electronic communication processes such as electronic mail, listservers, and online discussion boards, creates the opportunity to conduct "virtual ethics committee meetings" whereby information can be exchanged and interpretations of specific ethical case dilemmas put forward. This article discusses how that process can occur, relates our experience with such processes at two hospitals in our medical centre and considers some of the ethical and operational issues raised by such an approach.

Ethics committees are oriented toward developing consensus on clinical bioethical dilemmas. ${ }^{1}$
This reflects a pragmatic approach and is commonly associated with a consultation model that is instructional and communicative. ${ }^{2}$ Even traditional medical consultations contain some value judgments. ${ }^{3}$ However, since ethics consultation requires a prompt and individualised response, it is rare to get the full ethics committee's input prospectively on consultations.

We suggest it is generally preferable that such prospective input be made so that a diversity of views can be available to the ethics consultant or ethics consultation team when conducting the consultation. This is particularly valuable when there is a single ethics consultant responding to the request for a consultation. Moreover it lends greater institutional authority to the ethics consultation when others have been informed of and inform the consultation. Electronic computer-based communications make this possible now in a way not previously possible.

Electronic communication has a variety of health care applications today, and these are expanding rapidly. Electronic mail communications are occurring between patient and physician in individual medical practices, in internet-based websites, and between physicians in medical consultation. ${ }^{4-5}$ It is natural then to enquire whether electronic communication can enhance the ethical consultation process since communication and consensus are so much at the core of ethics consultation.

\section{Experience with electronic communications within ethics committees}

VA HOSPITAL EXPERIENCE

Experience at the Westside Division of the VA Chicago Healthcare System's ethics advisory committee with electronic communication began in 1996 and was assessed until January $1998 .^{6}$ Requests for ethics consultations were answered on the hospital computer system in the same fasion as requests for clinical consultations. The consultations were formulated and forwarded by e-mail to twelve committee members, comprising seven physicians, one social worker, one patient representative, a chaplain and two nurses. The average time to respond to a consultation request was 8.9 hours with $63 \%$ of the requests responded to within 1.5 hours. There was an average of $9.1 \pm 11.5$ electronic mail responses by committee members to each 
consultation. This includes some multiple responses from the same member. The major issues addressed were questions or conflicts about withdrawal of therapy or do-not-resuscitate orders $(36 \%)$; patient capacity to consent (26\%); surrogate/patient-physician disagreement about treatment $(20 \%)$; resource utilisation $12 \%$, and confidentiality $(4 \%)$.

The ethics consultant at this institution found this process lowered the barrier to timely input of other committee members, shifting the deliberative component of the ethics consultation in the direction of committee consensus and away from the individual consultant. This increased the likelihood that the consultant's recommendations were consonant with the communal values of that institution.

EXPERIENCE AT THE UNIVERSITY HOSPITAL University of Illinois at Chicago Hospital instituted concurrent electronic review of the ethics case consultations by the ethics consultation service team. This review allowed all team members access to information about the consultation as provided by the primary consultant by a listserver through the medical centre electronic mail system. Principally one author (LAS) provided the consultation service during that time with input from other members of the consultation team. Summaries of the consultations were posted to the listserver, which included four physicians, one lawyer, one chaplain and one philosopher.

In a nine-month period, 39 consultations were performed. There were 68 responses averaging 1.7 response per consultation, ranging from zero to nine per case. Routine cases stimulated low response while controversial ones elicited the greatest response. Response time ranged from less than one hour to two days. The most perplexing cases generally received the most prompt responses. Electronic responses modified the consultant's activity and recommendations in several cases, especially those dealing with particularly uncommon problems.

\section{ELECTRONIC ACCESSING PROCEDURES}

In addition to using an e-mail listserver, a commercially available software program for discussion posting and commentary can be used. We are now converting to this methodology which will permit archiving, further limit access to parties with specific access codes, and permit access from any remote internet site-meaning parties can participate even if they are out of town at the time. The discussion board bars access to any directory or search mechanism and requires a password for access. All recorded attempts to access the site are recorded by user and time, even failed attempts. The management of such a site involves monitoring access and archiving completed cases. There has been no security breach in our experience with either system.

\section{Discussion}

DYNAMICS

Ethics committee meetings are usually small group meetings that are subject to the social dynamics of small group interaction as described by Jonathan Moreno. ${ }^{7}$ Electronic communication can be expected to differ from the typical small group interaction by lacking communication via facial expression, intonation, body language, and other nonverbal content to such interaction. Although this difference has yet to be extensively studied, some observations have been made regarding internet group communication that may be relevant. Neil Postman observes that online groups do not develop a sense of reciprocal obligation. ${ }^{8}$ This shortcoming can be overcome in our application by the continuation of regular actual ethics committee meetings, which preserve the sense of reciprocal obligation. The moral effects of online communication remain largely unstudied at this time. ${ }^{9}$ However, Kiesler, Siegel and McGuire found that groups using e-mails were more likely to make shifts in decisions than in face-to-face encounters, possibly because the absence of face-to-face encounters minimised the influence of a group leader and other normative influences. ${ }^{10}$

Electronic communication among ethics committee members offers the opportunity for prospective review of consultation, a process made feasible by such a rapid communication device. Such an approach was inconceivable a few years ago. ${ }^{11}$

Ad hoc meetings usually take several days to arrange and vital time may pass without the needed inputs. While in the more routine cases this did not always change recommendations, being able to work through electronic communication was found to be particularly valuable in the unusual cases with atypical features. Trends in consultation can be monitored on the listserver and areas needing educational and quality initiatives may be more readily identified than during the scheduled meetings alone.

Electronic communication should be viewed as supplementary to regular committee meetings and by no means replaces them. Moreover there may still be occasions, albeit less frequently, where ad hoc meetings of the committee may still be needed.

\section{CONFIDENTIALITY AND ENCRYPTION}

Spielberg notes that electronic communication between physician and patients is increasing and altering the relationship, much as the advent of the telephone did in an earlier era. ${ }^{12}$ She notes the need to obtain informed consent, encrypt e-mails to maintain confidentiality, and develop guidelines on the usage of e-mail.

The American Medical Informatics Association has a guideline for e-mail communication between patient and physician. It calls for informing patients of what type of information will be communicated by e-mail and notes that encryption for this purpose is limited by patients not having the encryption software. ${ }^{13}$ 
The situation regarding ethics committees differs from that of communication between patient and physician. No consent is needed for committee members and ethics consultants to communicate with one another. However, caution regarding confidentiality is a major concern in ethics case discussions. When committee e-mail communications are not encrypted, we encourage various practices that protect confidentiality. First the committee e-mails do not mention the patient by name. Second, committee members are urged not to leave e-mails describing ethics consultations on computer screens unattended. Third, they are asked to limit the access to their e-mails by other parties. Use of an on line discussion board, as noted earlier, permits access only to identified parties and records access history.

\section{PRACTICAL ASPECTS}

The potential for misunderstanding exists, given the one-dimensional nature of e-mail communication. ${ }^{14}$ This can be minimised by simultaneous, interactive online connections but some limitation in communication via electronic media remains. Moreover the affective and normative aspects of the communication may be reduced by the lack of face-to-face encounters. Therefore we conclude that this type of communication should supplement not replace actual meetings of the ethics committee.

Reluctance to engage in e-communications may vary by group or individual. The duration of the group's existence and its interaction may influence willingness to engage in electronic communication. Members' level of trust in one another may be another factor because a record of comments may persist. Frequency of checking one's e-mail also could influence response rate and time.

Electronic communication permits access to the information at remote sites throughout the globe. It also keeps a record of the ideas exchanged, which means trends can be detected in consultations and problem areas identified: these can then become a focus of educational activity.

\section{THEORETICAL CONSIDERATIONS}

As electronic communication involves parties in new ways of communicating facts, values and judgments, it is important that we remain cognisant of McLuhan's adage that the "medium is the message". ${ }^{15}$ The process of e-mail communication could potentially modify the nature of decision making itself. How e-mail committee meetings will influence consensus building and ethical judgments remains to be assessed and comprehensively analysed. It is easy to imagine that its effects will be substantial. Continuing to have regularly scheduled ethics committee meetings, however, dampens the effect.
As we use and analyse this new communication technology, we need to be aware of the effects of the technologies themselves as well as of the information they disseminate so rapidly. In addition to confidentiality concerns, the professional roles of ethics consultants and ethics committees can be affected by the technologies. Reasoned study and analysis of these technological developments will be important to assure that their implementation is beneficial as well as effective. This latest communication advance promises to enliven ethical debate, contributing both a new process that speeds communication and a new subject matter for deliberation.

Arnold R Eiser, MD, FACP, is Chief, Section of Internal Medicine and Professor of Medicine and Medical Education, University of Illinois at Chicago. Stanley $G$ Schade, MD, is Professor of Medicine, Section of Hematology/Oncology, University of Illinois at Chicago. Lisa K Anderson-Shaw, DrPH, is Ethics Consultant, University of Illinois at Chicago, College of Medicine. Timothy Murphy, PhD, is Professor of Philosophy in Biomedical Science, Department of Medical Education, University of Illinois at Chicago.

\section{References}

1 Moreno JD. Deciding together: bioethics and moral consensus. New York: Oxford University Press, 1995.

2 Wear S, Katz P, Adrzejewski B. Development of an ethics consultation service. HEC Forum 1990;2:75- 87.

3 Foucalt M. Birth of the clinic: an archaeology of medical perception. NewYork: Vintage, 1973.

4 Robinson T, Patrick P, Eng TR, Gustafson D. An evidencebased approach to interactive health communication: a based approach to interactive health communication: a
challenge to medicine in the information age. Fournal of the American Medical Association 1998;280:1264-9.

5 Mandl K, Feit S, Pena BM, Kohane IS. Growth and determinants of access in patent e-mail and Internet use. Archives of Pediatrics and Adolescent Medicine 2000;154:508-11.

6 Schade S, Eiser A. The electronic ethics committee. fournal of General Internal Medicine 1998;13:91.

7 Wolff KH. The sociology of Georg Simmel. New York: Free Press, 1950.

8 Postman N. Technopoly: the surrender of culture to technology. New York: Vintage, 1993.

9 Galston W. Does the Internet strengthen technology? Philosophy Public Policy 2000; 19:1-8.

10 Kiesler S, Siegel J, McGuire TW. Social psychological aspects of computer-mediated communication. American Psychologist of computer-mediat

11 Kelly DF, Hoyt JW. Ethics consultation. Critical Care Clinics 1996;12:49-70.

12 Spielberg, A. On call and online: socio-historical, legal, and ethical implications of e-mail for the patient-physician relationship. Fournal of the American Medical Association 1998; 280:1353-9.

13 Kane B, Sands DZ. Guidelines for the clinical use of electronic mail with patients. Fournal of the American Medical Informatics Association 1998;5:104-11.

14 Borowitz S, Wyatt J. The origin, content, and workload of e-mail consultations. Fournal of the American Medical Association 1998;280:1321-4

15 McLuhan M. Understanding media. Cambridge, Mass: MIT Press, 1964. 